\title{
THE STRUCTURE OF A SPECIAL CLASS OF NEAR-RINGS
}

\author{
STEVE LIGH \\ (Received 27 October 1969; revised 8 December 1969) \\ Communicated by B. Mond
}

\section{Introduction}

It is well known that a Boolean ring is isomorphic to a subdirect sum of twoelement fields. In [3] a near-ring $(B,+, \cdot)$ is said to be Boolean if there exists a Boolean ring $(B,+, \wedge, 1)$ with identity such that $\cdot$ is defined in terms of,$+ \wedge$, and 1 and, for any $b \in B, b \cdot b=b$. A Boolean near-ring $B$ is called special if $a \cdot b=(a \vee x) \wedge b$, where $x$ is a fixed element of $B$. It was pointed out that a special Boolean near-ring is a ring if and only if $x=0$. Furthermore, a special Boolean near-ring does not have a right identity unless $x=0$. It is natural to ask then whether any Boolean near-ring (which is not a ring) can have a right identity. Also, how are the subdirect structures of a special Boolean near-ring compared to those of a Boolean ring. It is the purpose of this paper to give a negative answer to the first question and to show that the subdirect structures of a special Boolean nearring are very 'close' to those of a Boolean ring. In fact, we will investigate a class of near-rings that include the special Boolean near-rings and the Boolean semirings as defined in [8].

\section{Preliminaries}

A (left) near-ring is an algebraic system $(R,+, \cdot)$ such that

(i) $(R,+)$ is a group,

(ii) $(R, \cdot)$ is a semigroup,

(iii) $x(y+z)=x y+x z$ for all $x, y, z \in R$.

In particular, if $R$ contains a multiplicative semigroup $S$ whose elements generate $(R,+)$ and satisfy.

(iv) $(x+y) s=x s+y s$, for all $x, y \in R$ and $s \in S$, we say that $R$ is a distributively generated (d.g.) near-ring.

The most natural example of a near-ring is given by the set $R$ of mappings of an additive group (not necessary abelian) into itself. If the mappings are added by adding images and multiplication is iteration, then the system $(R,+, \cdot)$ is a 
near-ring. If $S$ is a multiplicative semigroup of endomorphisms of $R$ and $R^{\prime}$ is the subnear-ring generated by $S$, then $R^{\prime}$ is a d.g. near-ring. Other examples of d.g. near-rings may be found in [5].

An element $r$ of $R$ is right (anti-right) distributive if

$$
(b+c) r=b r+c r \quad((b+c) r=c r+b r)
$$

for all $b, c \in R$. It follows at once that an element $r$ is right distributive if and only if $(-r)$ is anti-right distributive. In particular, any element of a d.g. near-ring is a finite sum of right and anti-right distributive elements.

The kernels of near-ring homomorphisms are called ideals. Blackett [2] showed that $K$ is an ideal of a near-ring $R$ if and only if $K$ is a normal subgroup of $(R,+)$ that satisfied

(i) $R K \leqq K$ and

(ii) $(m+k) n-m n \in K$, for all $m, n \in R$ and $k \in K$.

\section{Subdirect sums of near-rings}

The theory of subdirect sum representation for rings carries over almost word for word to near-rings [4]. A nonzero near-ring $R$ is subdirectly irreducible if and only if the intersection of all the nonzero ideals of $R$ is nonzero. The nearring analogue of Birkhoff's [1] fundamental result for rings can be stated as follows.

THEOREM 3.1. [4] Every near-ring $R$ is isomorphic to a subdirect sum of subdirectly irreducible near-rings.

For a more detailed discussion of subdirect sums of near-rings, see [4]. By using the technique of subdirect sum representation, it was shown in [6] that every d.g. near-ring $R$ with the property that $x^{2}=x$ for all $x$ in $R$ is a Boolean ring.

\section{4. $\beta$-near-rings}

Definition 4.1. A near-ring $R$ is called a $\beta$-near-ring if for each $x$ in $R$, $x^{2}=x$ and $x y z=y x z$ for all $x, y, z \in R$.

EXAMPLE 4.2. Let $(R,+)$ be a nontrivial group. Define multiplication by $a \cdot b=b$ for all $a, b \in R$. Then $(R,+, \cdot)$ is a $\beta$-near-ring for which $\cdot$ is not commutative and $(R,+)$ need not be of characteristic two.

EXAMPLE 4.3. The Boolean semirings as defined in [8] are $\beta$-near-rings for which addition is commutative.

EXAMPLE 4.4. The special Boolean near-rings as defined in [3] are $\beta$-nearrings. 
It is easily seen that if a $\beta$-near-ring $R$ has a right identity, then $R$ is a Boolean ring. In fact, we have the following much stronger result.

THEOREM 4.5. Let $R$ be a near-ring with the property that $x^{2}=x$ for all $x$ in $R$ and has a right identity $e$. Then $R$ is a Boolean ring.

Proof. Since $e$ is right distributive, the equation

$$
(e+e)^{2}=e+e
$$

tells that $e+e=0$. If $x$ is in $R$, then

$$
x+x=x(e+e)=0 .
$$

Hence every element of $(R,+)$ is of order two and consequently $(R,+)$ is commutative.

Let $w$ be an arbitrary element in $R$. Then

yields that

$$
(e+w)^{2}=e+w
$$

$$
(e+w) e+(e+w) w=e+w .
$$

It follows that $(e+w) w=0$ for all $w \in R$. Moreover, $0 w+0 w w=0$ implies that $0 w(e+w)=0$. Thus $0 w(e+w) w=0 w$ implies that $0 w 0=0 w$ and hence $0=0 w$ for all $w \in R$.

To complete the proof we now show that $(R, \cdot)$ is commutative. This would mean that each element in $R$ is right distributive and hence $R$ is a (commutative) Boolean ring.

Let $a$ and $b$ be arbitrary elements of $R$. Then

$$
\begin{aligned}
(a b+b a)(a b+b a) & =a b+b a, \\
(a b+b a) a b+(a b+b a) b a & =a b+b a, \\
(a b+b a) a b & =(a b+b a)+(a b+b a) b a, \\
(a b+b a) a b & =(a b+b a)(e+b a) .
\end{aligned}
$$

Thus we have that

$$
\begin{aligned}
(a b+b a) a b b a & =(a b+b a)(e+b a) b a \\
& =(a b+b a) 0 \\
& =0 .
\end{aligned}
$$

It follows that

$$
(a b+b a) a b a b=0 b=0 .
$$

Similarly, expand $(a b+b a)(b a+a b)=a b+b a$ as above, we obtain that $(a b+b a) b a=0$. Consequently $a b+b a=0$. This completes the proof since every element of $(R,+)$ is of order two.

Note that Theorem 4.5 furnishes a negative answer to the first question mentioned in the introduction. 


\section{Subdirect structure of $\beta$-near-rings}

Definition 5.1. A near-ring $(R,+, \cdot)$ is said to be small if there is an element $e$ of $R$ such that $e$ is a left multiplicative identity and for all $x \neq e$ in $R$, either $x$ is a left identity or else $x y=0 y$ for all $y$ in $R$.

It is clear that any two-element field is a small near-ring but certainly not conversely. Now we are ready to state our result which compares the subdirect structures of a $\beta$-near-ring to those of a Boolean ring.

THEOREM 5.2. Every $\beta$-near-ring $R$ is isomorphic to a subdirect sum of subdirectly irreducible near-rings $R_{i}$ where each $R_{i}$ is either a two-element field or a small near-ring.

To facilitate the discussion on the proof of Theorem 5.2, we first prove a few lemmas which are of interest in their own right.

LEMMA 5.3. If $R$ is a subdirectly irreducible $\beta$-near-ring then $R$ has a left identity.

Proof. For each $x$ in $R$, let

$$
A_{x}=\{y \in R: x y=0\} .
$$

By straight forward calculations, keeping in mind that $x y z=y x z$ for all $x, y$, $z \in R$, one can easily verify that $A_{x}$ is an ideal of $R$. If $A_{x}=0$, then $x$ is a left identity since $x(x y-y)=0$ for all $y \in R$.

Now let

$$
N=\left\{x \in R: A_{x} \neq 0\right\}
$$

Suppose $N=R$. Let

$$
A=\bigcap_{x \in N} A_{x}
$$

Then $A$ is not zero since $R$ is subdirectly irreducible. But if $w \neq 0$ is in $A$, then $w^{2}=w=0$. Thus there exists an element $e$ in $R$ such that $A_{e}=0$ and hence $e$ is a left identity.

LEMMA 5.4. If $R$ is a subdirectly irreducible $\beta$-near ring and if $z \neq 0$ such that $A_{z} \neq 0$, then $z y=0 y$ for all $y \in R$.

Proor. Since $A_{z} \neq 0$, it follows that $z \in N$ as defined in (2). Since $A \neq 0$, let $w \neq 0$ be an element in $A$. Thus $x w=0$ for all $x \in N$. If $w y=0$ for some $y \neq 0$ in $R$, then $w \in N$ and $w^{2}=w=0$, which is a contradiction. It follows that $w y \neq 0$ for any $y \neq 0$ in $R$. This means that $A_{w}=0$ and hence $w$ is a left identity. Thus

$$
z y=z w y=0 y \text { for all } y \in R .
$$

LEMMA 5.5. If $R$ is a subdirectly irreducible $\beta$-near-ring with the property that $0 y=0$ for all $y$ in $R$, then each $x \neq 0$ in $R$ is a left identity. 
Proof. Let $N$ be the set as defined in (2). Then Lemma 5.4 implies that if there exists an element $z \neq 0$ in $R$ such that $A_{z} \neq 0$, then $z y=0 y$ for all $y$ in $R$. In particular, $z z=0 z=0$. This contradiction implies that $N=0$. Hence each nonzero element in $R$ is a left identity.

LEMMA 5.6. If $R$ is a subdirectly irreducible $\beta$-near-ring with a nonzero right distributive element $r$, then $R$ is the two element field.

Proof. Since $r$ is right distributive, it follows that $0 r=0$. From Lemma 5.4 and (3) with $z=r$ and $y=r$, we see that $A_{r}=0$. Now let

$$
L_{r}=\{y \in R: y r=0\} \text {. }
$$

Since $r$ is right distributive and $x y z=y x z$ for all $x, y, z \in R$, it is easily verified that $L_{r}$ is an ideal of $R$. Suppose that $L_{r} \neq 0$. Let

$$
L=L_{\mathrm{r}} \cap A \text {, where } A=\bigcap_{x \in N} A_{x} .
$$

There exists a $w \neq 0$ in $L$ such that $x w=0$ for each $x \in N$ and $w r=0$. This is a contradiction since $w$ is a left identity. Thus $L_{r}=0$ and we conclude that $r$ is a right identity as well as a left identity. Thus $(R, \cdot)$ is commutative and $0 x=0$ for all $x$ in $R$. By Lemma 5.5 each $x \neq 0$ in $R$ is a left identity and it follows that $x=x r=r$. Consequently $R$ is the two-element field.

We may now complete the proof of Theorem 5.2.

Proof of Theorem 5.2. Let $R$ be a $\beta$-near-ring. By Theorem 3.1, $R$ is isomorphic to a subdirect sum of subdirectly irreducible near-rings $R_{i}$. Now each $R_{i}$ is a homomorphic image of $R$ and therefore a $\beta$-near-ring. If $R_{i}$ has a nonzero right distributive element then it is a two-element field by Lemma 5.6. If $R_{i}$ does not have a nonzero right distributive element, then $R_{i}$ is a small near-ring by Lemmas 5.3 and 5.4 .

Since special Boolean near-rings and Boolean semi-rings as defined in [3] and [8] respectively are $\beta$-near-rings, Theorem 5.2 furnishes the subdirect structures of those near-rings as well.

An immediate corollary of Lemma 5.6 is the following characterization of Boolean rings.

COROLlary 5.7. A near-ring $R$ is a Boolean ring if and only if $R$ is a $\beta$-nearring and every nonzero homomorphic image of $R$ has a nonzero right distributive element.

Since a homomorphic image of a d.g. near-ring is again a d.g. near-ring [5], we have

COROLlaRY 5.8. Every d.g. $\beta$-near-ring is a Boolean ring. 


\section{Remarks}

In view of Theorem 4.5, one naturally asks that if $R$ is a near-ring with a right identity, for what positive integers $n$ such that $x^{n}=x$ for all $x$ in $R$ would imply that $(R, \cdot)$ is commutative. Of course it is well known that if $R$ is a ring, then $(R, \cdot)$ is commutative for all $n$. By a result in [7, Cor. 3.7] an affirmative answer for $n=n_{0}$ would imply that if $R$ is a near-ring with a right identity and $x^{n_{0}}=x$ for all $x$ in $R$, then $R$ is a commutative ring with identity. Thus it is of interest to known the answers to the questions just mentioned above.

\section{References}

[1] G. Birkhoff, 'Subdirect unions in universal algebra', Bull. Amer. Math. Soc. 50 (1944), $764-768$.

[2] D. W. Blackett, 'Simple and semisimple near-rings', Proc. Amer. Math. Soc. 4 (1953), $772-785$.

[3] J. R. Clay and D. A. Lawver, 'Boolean near-rings', Canad. Math. Bull 12 (1969), 265-274.

[4] C. G. Fain, Some structure theorems for near-rings. Doctoral Dissertation, University of Oklahoma, 1968.

[5] A. Frohlich, 'Distributively generated near-rings. I. Ideal Theory, Proc. London Math. Soc. 8 (1958), 76-94.

[6] S. Ligh, 'On Boolean near-rings', Bull. Australian Math. Soc. 1 (1969), 375-380.

[7] S. Ligh, 'On regular near-rings', (to appear).

[8] N. V. Subrahmanyam, 'Boolean semirings', Math. Ann. 148 (1962), 395-401.

Department of Mathematics

University of Florida

Gainesville, Florida 32601 